\title{
Fas and Fas ligand expression in cystic fibrosis airway epithelium
}

Isabelle Durieu, Carole Amsellem, Christian Paulin, Marie-Thérèse Chambe, Jacques Bienvenu, Gabriel Bellon, Y Pacheco

\begin{abstract}
Background-Cystic fibrosis (CF) is a genetic disease caused by mutations in the cystic fibrosis transmembrane conductance regulator (CFTR) gene and defective expression of CFTR protein in epithelial cells. The main cause of mortality in $\mathrm{CF}$ is linked to chronic inflammatory and infectious airway processes. Recent studies have suggested perturbations in the apoptotic process in CF cell lines and enterocytes. A study was undertaken to investigate the expression of Fas and Fas ligand (FasL) in CF bronchial epithelium and CF tracheal cell lines.
\end{abstract}

Methods-Immunohistochemical staining for Fas (alkaline phosphatase anti-alkaline phosphatase) and FasL (immunoperoxidase) was performed in eight CF bronchial epithelial samples and four controls and immunohistochemical DNA fragmentation (TUNEL) was carried out in four CF patients and four controls. Immunofluorescence staining and flow cytometric analysis of Fas and FasL expression was performed in two human tracheal epithelial cell lines (HTEC) with normal and CF genotype. The dosage of serum soluble FasL was examined in 21 patients with $\mathrm{CF}$ and 14 healthy volunteers.

Results-FasL expression was markedly increased in patients with CF in both the ciliated and submucosal glandular bronchial epithelium compared with controls; Fas was similarly expressed in bronchial samples from controls and CF patients in both the ciliated epithelium and submucosal glands. High levels of DNA fragmentation were observed in CF but with some epithelial cell alterations. Serum concentrations of soluble FasL were frequently undetectable in patients with $\mathrm{CF}$. In vitro, HTEC expressed Fas and FasL in both genotypes. A higher mean fluorescence intensity for FasL expression was noted in CF genotype HTEC with median (range) for six experiments of 74 (25-101) for CF cells and 42 (21-70) for non-CF cells. Conclusion-Fas/FasL interaction is probably implicated in the human CF airway apoptotic pathway. The mechanisms of induction of FasL expression and its role in inducing tissue damage or remodelling or in controlling local inflammatory cell apoptosis remain to be determined. (Thorax 1999;54:1093-1098)

Keywords: cystic fibrosis; apoptosis; Fas/APO1; Fas ligand
Cystic fibrosis (CF) is a genetic disease caused by mutations in the cystic fibrosis transmembrane regulator (CFTR) gene resulting in absent or deficient expression and function of CFTR protein. ${ }^{12}$ The main cause of morbidity and mortality in $\mathrm{CF}$ is linked to a chronic inflammatory and infectious airway process which leads to progressive bronchial epithelial damage and lung destruction. $^{34}$

Defective electrolyte transport is believed to be the initial abnormality in CF disease resulting in alteration of the epithelial secretions. Evidence of an early and intense airway inflammation, probably even in the absence of documented infection, has been reported in several studies. ${ }^{56}$ A possible role of CFTR dysfunction in the induction and maintenance of the inflammatory and infectious process through a dysregulation of epithelial ion transport and abnormal extracellular fluid has been suggested. ${ }^{78}$ It has recently been reported that CFTR may also be involved in the apoptotic process of epithelial cells. ${ }^{9} 10$ Apoptosis is a physiological process essential for the maintenance of homeostasis of epithelial organisation and function, and for clearance of inflammatory cells. Apoptosis is regulated by several factors including oxidative stress, ${ }^{11}$ extracellular matrix proteins, ${ }^{12}$ and external signals such as Fas ligation. ${ }^{13}$ Fas receptor (CD95/APO1) belongs to the tumour necrosis factor (TNF) receptor superfamily. It is commonly expressed on lymphocytes as well as on the cell surface of various epithelial cells mediating interactions with immune effector cells. ${ }^{14}$ Fas receptor activation by its ligand (Fas ligand) induces apoptosis of Fas bearing cells. Fas ligand (FasL) belongs to the TNF family and is predominantly expressed on activated $\mathrm{T}$ cells. ${ }^{15}$ The expression of FasL in sites such as the eye and in some human carcinomas contributes to the existence of "immune privilege" of these tissues by inducing apoptosis in Fas bearing immune cells. ${ }^{16}$ Its expression in epithelial tissue suggests a role for FasL in controlling epithelial tissue injury during various inflammatory states. ${ }^{18}$ As described for TNF- $\alpha$, there is a soluble form of FasL obtained by conversion of membrane bound FasL through a proteolytic process and devoid of apoptotic activity. ${ }^{19}$

In this study we examined (1) the epithelial expression of Fas and Fas ligand in bronchial samples obtained from CF patients and non-CF patient controls; (2) the expression of Fas/FasL in two human tracheal epithelial cell 
lines, one of them with a CF genotype and the other with the normal CFTR genotype; and (3) the serum concentrations of soluble FasL in patients with CF and healthy controls.

\section{Methods}

IMMUNOHISTOCHEMICAL STUDY

Patients

Between 1993 and 1997 eight patients with CF (2M:6F) of mean age 16 (range 6-30) underwent bronchial biopsies (five patients) or lobectomy (three patients) because of repeated infectious complications with localised bronchiectasis. Patient characteristics and recruitment have been previously described. ${ }^{20}$ Four patients carried $\Delta \mathrm{F} 508 / \Delta \mathrm{F} 508$ mutations and four $\Delta \mathrm{F} 508$ /other mutations. Forced expiratory volume in one second $\left(\mathrm{FEV}_{1}\right)$ varied from $26 \%$ to $86 \%$ predicted and forced vital capacity (FVC) from 32\% to $99 \%$ predicted. Two patients had chronic sputum colonisation with Staphylococcus aureus, the others with Pseudomonas aeruginosa.

Four male subjects who underwent lobectomy for bronchial carcinoma were used as controls using non-neoplastic bronchial samples.

\section{Bronchial tissues}

The fibreoptic bronchial biopsy specimens and selected samples of bronchi obtained from surgical lobectomies were embedded in OCT compound (Tissue Tek), snap frozen in liquid nitrogen, and stored at $-80^{\circ} \mathrm{C}$ until cryosectioning.

\section{Immunohistochemistry: antigen expression and detection of DNA fragmentation}

Five $\mu \mathrm{m}$ cryostat tissue sections were mounted onto poly-L-lysine coated slides and stored at $-20^{\circ} \mathrm{C}$. The slides were air dried for 30 minutes and fixed in $4 \%$ paraformaldehyde for $30 \mathrm{~min}$ utes. Tissue sections were incubated with antiFas monoclonal antibody (UB2, Immunotech SA, Marseille, France) at a 1:500 dilution in PBS/Triton $2 \%$ for one hour at room temperature. PBS/Triton $2 \%$ was substituted for the primary antibody for the negative controls. Samples were then washed extensively in Tris buffer $0.1 \mathrm{M}$ and incubated for 30 minutes with a rabbit anti-mouse secondary antibody at a dilution of 1:25. After washing, samples were incubated with a mouse monoclonal alkaline phosphatase anti-alkaline phosphatase antibody (Sigma Immuno Chemicals, St Louis, Missouri, USA) at a dilution of 1:50 for 30 minutes sheltered from light. Detection of antibody was visualised with the Fast ${ }^{\mathrm{TM}}$ Fast Red TR naphthol substrate (Sigma Immuno Chemicals). Tissue sections incubated with mouse monoclonal alkaline phosphatase antialkaline phosphatase antibody alone served as negative controls.

For FasL staining, tissue sections were incubated for 30 minutes in $\mathrm{H}_{2} \mathrm{O}_{2} \quad 0.3 \%$ washed in Tris buffer and incubated with anti-FasL rabbit polyclonal antibody (SC956; Santa Cruz Biotechnologies, Santa Cruz, California, USA) at a dilution of 1:100 for one hour. After washing a peroxidase conjugated secondary antibody at a 1:500 dilution was applied for one hour. Detection of antibody was visualised with 3,3'diaminobenzidine in Tris buffer. Tissue sections incubated with the peroxidase conjugated secondary antibody served as negative control. FasL antibody was used in the presence or absence of the corresponding blocking peptide (amino acid residues 2-19, Santa Cruz Biotechnologies) to confirm the specificity of the staining.

To detect DNA fragmentation in bronchial epithelium the terminal deoxynucleotidyl transferase (TdT) mediated dUTP nick end labelling (TUNEL) method using an in situ cell death detection kit, fluorescein, and peroxidase antifluorescein antibody (Boehringer Mannheim) was used in four patients with $\mathrm{CF}$ and controls.

FAS AND FAS LIGAND EXPRESSION IN AIRWAY CELI LINES

\section{Cultures of cell lines}

Two human fetal SV40 transformed tracheal epithelial cell (HTEC) lines were studied-one with the CF genotype CFT-2 homozygous for
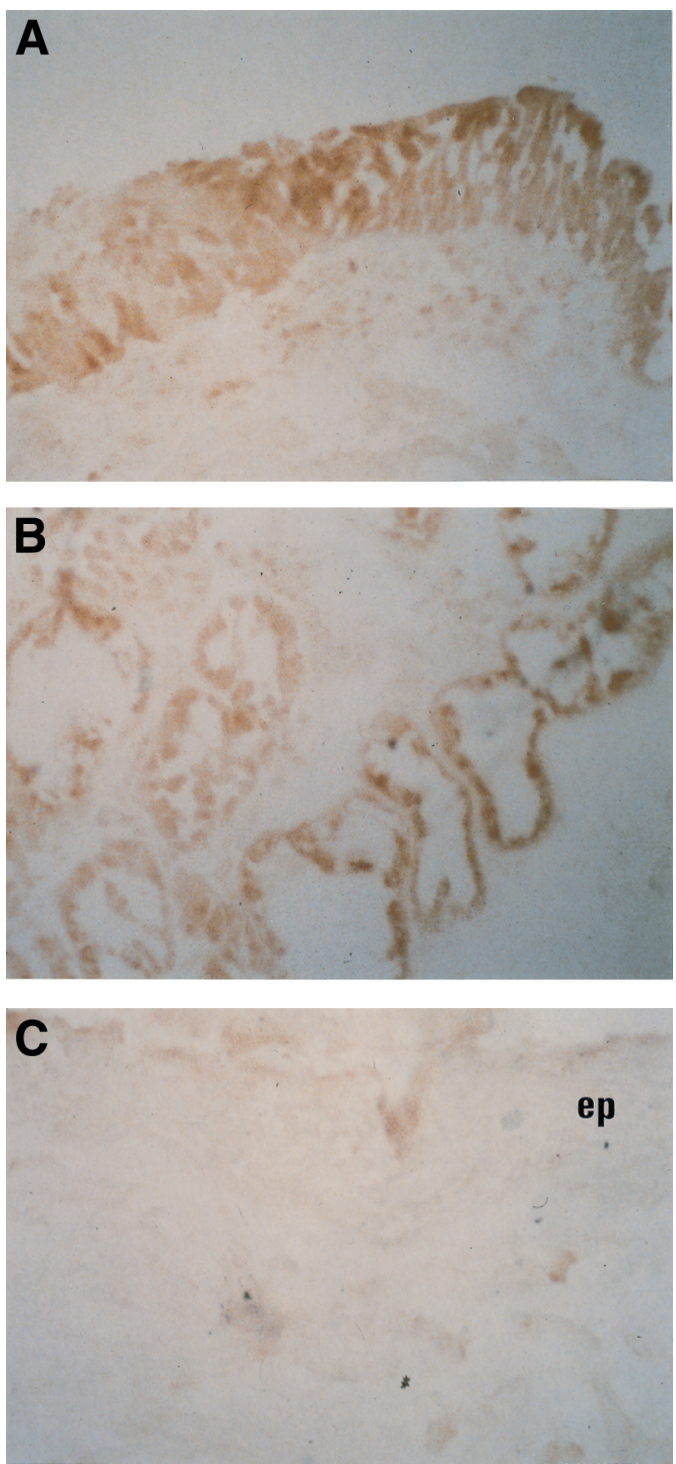

Figure 1 Fas ligand immunoperoxidase staining showing diffuse staining in $(A)$ the ciliated epithelium and $(B)$ the glandular area of a patient with $C F$ and $(C)$ very low epithelial (ep) staining in control patient. Magnification $\times$ 20 for $A$ and $B, \times 40$ for $C$. 

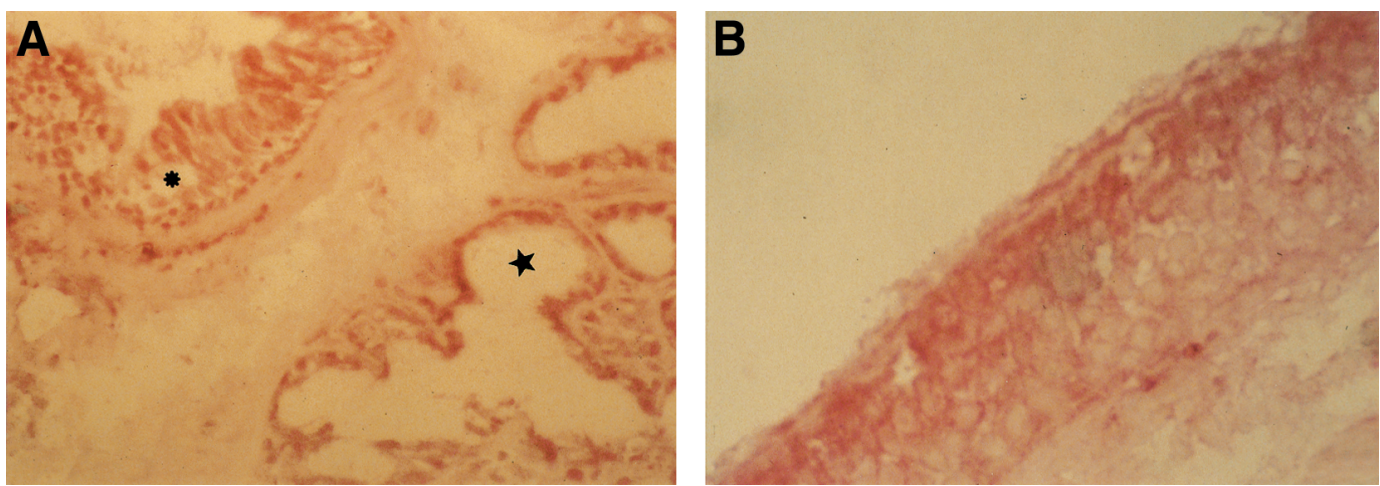

Figure 2 Fas alkaline phosphatase anti-alkaline phosphatase immunostaining showing $(A)$ positive staining in both ciliated (*) and glandular epithelium (*) of patients with CF and (B) positive staining in control patient. Magnification $\times 20$ for $A, \times 40$ for $B$.

$\Delta \mathrm{F} 508$ mutations and one wild CFTR genotype cell line (NT) used as a control. ${ }^{21}$ The two HTEC lines were cultured in DMEMHAMF12 medium (Gibco) supplemented with $10 \%$ fetal calf serum (Gibco) and $1 \%$ penicillin/streptomycin/fungizon (Sigma) at $37^{\circ} \mathrm{C}$ in a $5 \% \mathrm{CO}_{2}$ atmosphere.

Immunofluorescence staining and flow cytometric analysis

Surface antigen expression was studied in unstimulated HTEC lines and in HTEC lines treated with TNF- $\alpha(100 \mathrm{U} / \mathrm{ml}$, Sigma) or interferon gamma (IFN- $\gamma)(1000 \mathrm{U} / \mathrm{ml}$, Genzyme) for 48 hours before immunofluorescence staining. The cells were removed from the dish with a solution containing versen and then washed in PBS. Mouse anti-human Fas monoclonal antibody $(5 \mu \mathrm{g} / \mathrm{ml}$, UB2, Immunotech SA) and rabbit anti-human FasL polyclonal antibody (SC956, $10 \mu \mathrm{g} / \mathrm{ml}$, Santa Cruz Biotechnologies) were incubated with cells on ice for 45 minutes with concomitant permeabilisation with saparin for FasL staining. After washing with $\mathrm{PBS} / 1 \%$ bovine serum albumin the cells were labelled with R-phycoerythrin (RPE) conjugated anti-mouse secondary antibody at a 1:100 dilution for Fas staining and fluorescein isothiocyanate (FITC) conjugated anti-rabbit antibody at a dilution of 1:50 for FasL staining. Cells were incubated in the dark for 45 minutes on ice, washed with PBS and resuspended before analysis by fluorescence activated cell sorting (FACS EPICS XL-MCL, Coulter). RPE and FITC conjugated alone and irrelevant isotype matched anti-human immunoglobulin served as negative controls. The

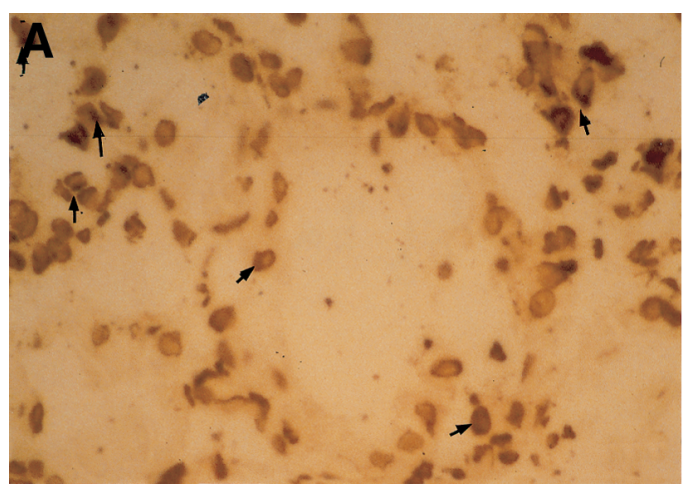

mean (SD) fluorescence intensity was analysed. Six experiments were performed for statistical analysis for each antigen ( $F a s$ and FasL), each condition (basal condition and cytokine pretreatment), and each cell line (NT and CFT-2). The Wilcoxon signed rank test was used to compare the mean fluorescence intensity at basal conditions between NT and CFT-2 for Fas and FasL and the mean fluorescence intensity for Fas and FasL expression between basal and stimulated cells (IFN- $\gamma$ or TNF- $\alpha$ pretreatment) for each cell line.

MEASUREMENT OF SERUM SOLUBLE FAS LIGAND Serum soluble FasL (sFasL) was measured in 21 successive patients with CF and 14 normal subjects (blood donor serum samples). Measures were obtained with the FasLigand ELISA kit (MBL Co Ltd, Nagoy, Japan). FasL was measured by sandwich ELISA. Samples were incubated in wells coated with the anti-FasL monoclonal antibody 4H9 for one hour. After washing, a peroxidase conjugated anti-FasL monoclonal antibody (4A5) was added and incubated for a further hour. An acid solution was then added to terminate the enzyme reaction and to stabilise the developed colour. The optical density (OD) of each well was measured at $450 \mathrm{~nm}$ using a microplate reader. The concentration of sFasL was calibrated from a dose response curve based on reference standards with a threshold detection concentration of $0.03 \mathrm{ng} / \mathrm{ml}$. Because of possible undetectable values a Yates' $\chi^{2}$ test was used to compare the partition of serum soluble FasL concentrations between CF patients and healthy controls.

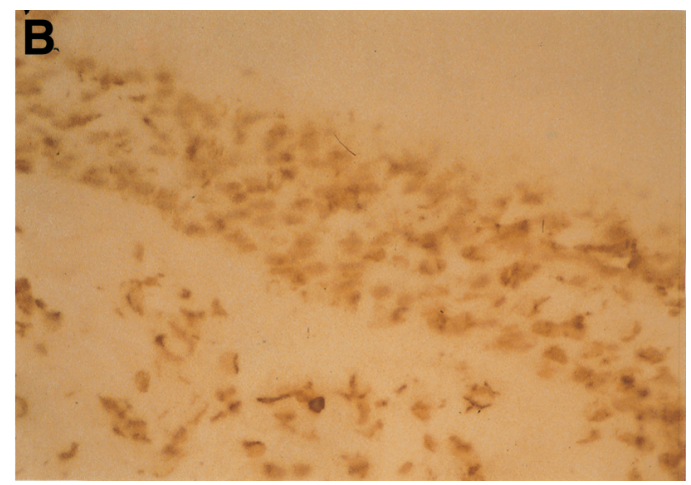

Figure 3 TUNEL immunostaining showing (A) intranuclear DNA fragmentation (arrows) in submucosal glandular area of patient with $C F$; $(B)$ control. Magnification $\times 40$ for $A, \times 20$ for $B$. 


\section{Results}

IMMUNOHISTOCHEMISTRY

FasL was expressed with high intensity in all patients with $\mathrm{CF}$, both in the ciliated epithelium and in the submucosal glandular epithelium, with diffuse cytoplasmic staining (fig 1A and $\mathrm{B}$ ). Both unistratified and epidermoid metaplastic epithelium gave an intense staining compared with controls (fig 1C). Fas antibody gave a membranous bronchial epithelial staining in normal and metaplastic epithelium and in submucosal glands, both in patients with $\mathrm{CF}$ and in controls, with no significant difference between them (fig 2A and B). DNA fragmentation with intense intranuclear staining using TUNEL method was observed in some bronchial epithelial cells and submucosal glands of all CF samples but not in controls (fig $3 \mathrm{~A}$ and $\mathrm{B}$ ). However, the $\mathrm{CF}$ epithelium was not always well preserved and the interpretation of such a difference is difficult.

ANTIGEN EXPRESSION ON CULTURE CELL LINES The mean fluorescence intensities for Fas and FasL staining measured by flow cytometric analysis are summarised in table 1. Both NT and CF-T2 cells expressed Fas with no significant difference in the mean intensity of fluorescence $(p=0.11)$. In both cell lines Fas expression was not modified after stimulation by IFN- $\gamma$ and TNF- $\alpha$. The mean fluorescence intensity of FasL staining was significantly higher for CFT-2 $(\mathrm{p}=0.04)$ and pretreatment with TNF- $\alpha$ or IFN- $\gamma$ did not have any significant effect. The apparent difference in FasL expression observed between NT and CF-T2 after IFN- $\gamma$ was not statistically significant $(\mathrm{p}=$ 0.07).

SERUM SOLUBLE FAS LIGAND

The serum concentration of FasL was very low in CF patients with undetectable concentrations $(<0.03 \mathrm{ng} / \mathrm{ml})$ in 13 of 21 patients. All the normal subjects had detectable concentrations of sFasL ranging from 0.08 to $0.38 \mathrm{ng} / \mathrm{ml}$ $\left(p<0.001\right.$,Yates' $\chi^{2}$ test $)$. The median concentration was $0.125 \mathrm{ng} / \mathrm{ml}$ for normal subjects and was below the threshold detection value for patients with CF (fig 4). The undetectable concentrations were not associated with therapeutic drugs, particularly corticosteroids.

Table 1 Mean fluorescence intensities for Fas ligand and Fas staining in human SV40-transformed tracheal epithelial cell lines with CF (CFT-2) and normal (NT) genotype (fluorescence activating cell sorting). Measures are expressed for unstimulated cells (basal) and after stimulation with tumour necrosis factor- $\alpha$ (TNF- $\alpha$ ) and interferon- $\gamma$ $(I F N-\gamma)(n=6)$

\begin{tabular}{clllll}
\hline & & Basal & TNF & IFN & p value \\
\hline Fas ligand & Median & 74 & 66 & 50 & NS \\
CFT-2 & Range & $25-101$ & $51-77$ & $24-71$ & NS \\
NT & Median & 42 & 37 & 38 & \\
p value & Range & $21-70$ & $23-67$ & $21-79$ & \\
Fas & & 0.04 & & & \\
CFT-2 & Median & 38 & 20 & 33 & NS \\
NT & Range & $13-45$ & $14-43$ & $14-52$ & NS \\
p value & Median & 21 & 18 & 22 & \\
& Range & $16-36$ & $14-24$ & $16-48$ & \\
\hline
\end{tabular}

NS $=$ non-significant difference $(p>0.05)$.

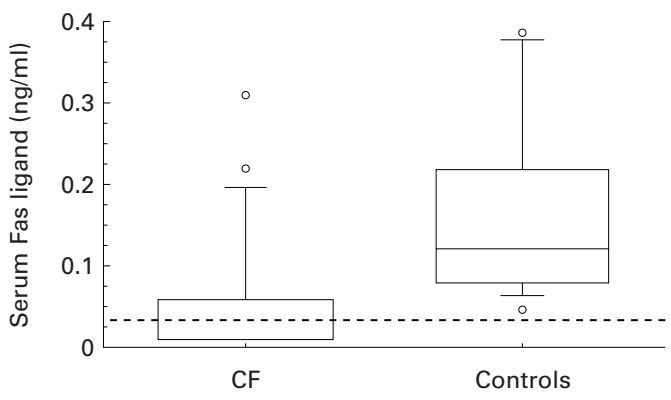

Figure 4 Serum concentration of soluble Fas ligand in 21 patients with cystic fibrosis and 14 healthy controls. Threshold detectable concentration $0.03 \mathrm{ng} / \mathrm{ml}$.

\section{Discussion}

Our results show that in vivo FasL expression is markedly intense in human CF bronchial epithelium compared with controls; Fas is expressed in both CF and control bronchial epithelium with a similar intensity. More intense DNA fragmentation is also observed in patients with CF relative to controls but sometimes in impaired epithelium. In the cultures both deficient and normal CFTR genotype human tracheal cell lines expressed Fas and FasL but the mean fluorescence intensity for FasL expression was higher in CF genotype cells. Cytokine stimulation did not modify Fas and FasL expression in the two cell lines examined. Serum soluble Fas ligand is unmeasurable or is present in very low concentrations in most patients with CF.

Our results are consistent with recent publications showing expression of both Fas and FasL in human airway epithelium. ${ }^{22}{ }^{23}$ However, in our study the immunohistochemical staining for FasL is intense in patients with $\mathrm{CF}$ but very low in controls. The results of HTEC expression are in agreement with immunohistochemical findings, showing a significantly increased expression of FasL in the CF genotype cell line. On the other hand, Fas expression is the same in both human tissue and HTEC lines.

Apoptosis dysfunction in cystic fibrosis has recently been suggested with varying results. Gottlieb et al reported a "resistance" in vitro in the initiation of apoptosis in a mutant CFTR epithelial mouse mammary cell line caused by an inability to obtain cytoplasmic acidification compared with the wild CFTR genotype cell line. ${ }^{9}$ On the other hand, Maiuri et al observed increased DNA fragmentation ${ }^{10}$ and increased Fas and FasL expression in CF enterocytes suggesting a possible role for Fas ligation in the induction of apoptosis in CF enterocytes. ${ }^{24}$

The high level of FasL staining observed in vivo in CF bronchial epithelium suggests various hypotheses. Firstly, the implication of local and chronic inflammation in the induction of FasL expression: stimulation by cytokines in this study and others did not increase Fas and FasL expression ${ }^{25}$ but other cytokines such as IL-8 present in CF airways could be implicated in vivo. ${ }^{5}$ Secondly, normal bronchial epithelial cells are dependent on cell-matrix interactions to prevent apoptosis ${ }^{26}$; moreover, extracellular matrix proteins can induce Fas expression in epithelial cells. ${ }^{27}$ The major modifications in 
the organisation of the extracellular matrix in $\mathrm{CF}$, with subepithelial fibrosis and degradation of collagenic and elastic fibres, ${ }^{20}$ may have an important role in controlling cell apoptosis and membranous expression of proteins involved in the regulation of cell turnover and tissue remodelling in CF. Thirdly, modification of FasL expression may be related to dedifferentiation of the airway surface epithelium and epidermoid metaplasia frequently observed in CF. However, FasL expression was not intense in some metaplastic areas of control samples obtained from lobectomies for bronchial carcinoma.

The high level of FasL expression in CF genotype tracheal cells compared with wild genotype cells suggests a role for deficient CFTR in inducing FasL expression. The modification of chloride secretion and cellular polarity, the accumulation of mutant CFTR in endoplasmic reticulum, and the upregulation of IL-8 expression in CF bronchial cells could induce the activation of transcriptional factors such as $\mathrm{NK}-\kappa \mathrm{B}$. NF- $\kappa \mathrm{B}$ plays an important role in the regulation of inducible genes such as IL-8, TNF- $\alpha$ and FasL. ${ }^{28-30}$

The significance of low levels of sFasL observed in patients with CF is unclear. The soluble trimeric form of FasL results from a metalloproteinase mediated proteolytic process in the extracellular domain of membrane bound FasL. ${ }^{19}$ To date, high levels of sFasL and soluble Fas have been described in haematopoietic and non-haematopoietic human cancer, in autoimmune disease, in viral hepatitis, and in alcoholic liver disease. ${ }^{31-33}$ Processing and release represent a downregulation of proapoptotic activity of membrane bound ligand. ${ }^{19}{ }^{34}$ Undetectable levels of the soluble form have not been described in other diseases; our results could suggest the maintenance of apoptotic activity.

The consequences of the expression of both Fas and FasL in the same epithelium are unclear. Similar findings have been reported recently in autoimmune diseases such as Hashimoto's thyroiditis and primary Sjörgen's syndrome, while cells from normal individuals only express Fas. ${ }^{356}$ These results suggest that in autoimmune diseases a suicidal or fratricidal programmed cell death involving a Fas/FasL interaction occurs leading to epithelial cell destruction. A similar implication in $\mathrm{CF}$ epithelium remains to be demonstrated. Other studies in animal models and asthmatic patients suggest a potential role for FasL in modulating bronchial inflammation inducing apoptosis in immune cells during inflammatory reactions. ${ }^{1822} 23$ FasL expression may also play a part by maintaining an "immune privileged" status in sites such as the ey $\mathrm{e}^{16}$ and by conferring immune suppression in malignancy ${ }^{17}$ inducing apoptosis of Fasbearing immune cells such as lymphocytes and dendritic cells. Another recent study showed that FasL also has pro-inflammatory effects since it can stimulate polymorphonuclear neutrophils (PMNs); activated PMNs directly mediate cytolysis of FasL+ cells. ${ }^{37}$ In CF the possible apoptotic interaction between PMNs and FasL+ airway epithelial cells is important since PMNs are the predominant phagocytes in bronchial secretions even in patients with clinically mild lung disease. ${ }^{5}$ On the other hand, PMNs express both Fas and FasL and activation of the Fas pathway by Fas-FasL interaction is one of the mechanisms of PMN apoptosis. ${ }^{38}$ PMNs are the major source of the increased quantity of DNA found in CF sputum which essentially contributes to the altered viscosity of CF airway secretions. ${ }^{3940}$ Another hypothesis could be that FasL+ CF airway epithelial cells activate Fas mediated apoptosis of PMNs thereby inducing enhanced DNA release and alterations in mucus viscoelasticity.

The mechanisms of induction of FasL expression in CF airway epithelial cells remain to be determined. Whether it contributes to airway tissue damage or to the clearance of inflammatory cells is not yet known.

The authors thank N Billard, M Gutowski and C Dumontet for their technical contribution.

The study was funded by a grant from Hospices Civils de Lyon.

1 Riordan JR, Rommens JM, Kerem B, et al. Identification of the cystic fibrosis gene: cloning and characterization of complementary DNA. Science 1989;245:1066-73

Welsh MJ, Anderson MP, Rich DP, et al. Cystic fibrosis, CFTR and abnormal electrolyte transport. In: Davis PB, ed. Cystic fibrosis. New York: Marcel Dekker, 1993: 29-52.

3 Davis PB. Pathophysiology of the lung disease in cystic fibrosis. In: Davis PB, ed. Cystic fibrosis. New York: Marcel Dekker, 1993: 193-218.

4 Ogrine G, Kampalath B, Tomashefski JF. Destruction and loss of bronchial cartilage in cystic fibrosis. Human Pathol 1998;29:65-73.

5 Khan TZ, Wagener JS, Bost T, et al. Early pulmonary inflammation in infants with cystic fibrosis. Am f Respir Crit inflammation in infants with cyst

6 Zahm JM, Gaillard D, Dupuit F, et al. Early alteration in airway mucociliary clearance and inflammation of the lamina way mucociliary clearance and inflammation of the

7 Goldman MJ, Mark Anderson G, Stolzenberg ED, et al. Human $\beta$-defensin- 1 is a salt-sensitive antibiotic in lung that is inactivated in cystic fibrosis. Cell 1997;88:553-60.

8 Tabary O, Zahm JM, Hinnrasky J et al. Selective up-regulation of chemokine IL-8 expression in cystic fibrosis bronchial gland cells in vivo and in vitro. Am 7 Pathol 1998;153:921-30.

9 Gottlieb RA, Dosanjh A. Mutant cystic fibrosis transmembrane conductance regulator inhibits acidification and apoptosis in C127 cells: possible relevance to cystic fibrosis. Proc Natl Acad Sci USA 1996;93:3587-91.

10 Maiuri L, Raia V, De Marco G, et al. DNA fragmentation is a feature of cystic fibrosis epithelial cells: a disease with inappropriate apoptosis? FEBS 1997;408:225-31.

11 Buttke TM, Sandstrom PA. Oxidative stress as a mediator of apoptosis. Immunol Today 1994;15:7-10.

12 Meredith JE, Fazeli JB, Schwartz MA. The extracellular matrix as a cell survival factor. Mol Biol Cell 1993;4 953-61.

13 Nagata S, Golstein P. The fas death factor. Science 1995;267:1449-56.

14 Leithaüseur F, Dhein J, Mechtersheimer G, et al. Constitutive and induced expression of APO-1, a new member of the nerve growth factor/tumor necrosis factor receptor superfamily, in normal and neoplastic cells. Lab Invest superfamily, in

15 Suda T, Nagata S. Purification and characterization of the Fas-ligand that induces apoptosis. F Exp Med 1994;179: Fas-ligand

16 Griffith TS, Brunner T, Flechter SM, et al. Fas ligandinduced apoptosis as a mechanism of immune tolerance. Science 1995;270:1189-92.

17 Niehans GA, Brunner T, Frizelle SP, et al. Human lung carcinomas express Fas ligand. Cancer Res 1997;57:1007-12.

18 Gochuico BR, Miranda KM, Hessel EM, et al. Airway epithelial Fas ligand expression: potential role in modulating bronchial inflammation. Am $\mathcal{f}$ Physiol 1998; 274:L444-9.

19 Schneider P, Holler N, Bodmer JL, et al. Conversion of membrane-bound Fas (CD95) ligand to its soluble form is associated with downregulation of its proapoptotic activity and loss of liver toxicity. f Exp Med 1998;187: activity and 1205 .

20 Durieu I, Peyrol S, Gindre D, et al. Subepithelial fibrosis and degradation of the bronchial extracellular matrix in cystic fibrosis. Am f Respir Crit Care Med 1998;158:580-8. 
21 Lemnaouar M, Chastre E, Paul A, et al. Oncogene-mediated propagation of tracheal epithelial cells from two cystic fibrosis fetuses with different mutations. Characterization of CFT- 1 and CFT-2 cells in culture. Eur $\mathcal{f}$ Clin Invest 1993;23:151-60

22 Hamann KJ, Dorscheid DR, Ko FD, et al. Expression of Fas CD95) and FasL (CD95L) in human airway epithelium. Am f Respir Cell Mol Biol 1998;19:537-42.

23 Druilhe A, Wallaert B, Tsicopoulos A, et al. Apoptosis, proliferation, and expression of Bcl-2, Fas and Fas ligand in bronchial biopsies from asthmatics. Am 7 Respir Cell Mol Biol 1998;19:747-57.

24 Maiuri L, Raia V, De Marco G, et al. Involvement of Fas and Fas-ligand in cystic fibrosis. XXth European CF Conference. Berlin: June 1998 (abstract).

25 Wen LP, Madani K, Fahrni JA, et al. Dexamethasone inhibits lung epithelial cell apoptosis induced by IFN- $\gamma$ and Fas.

26 Aoshiba K, Rennard SI, Spurzem JR. Cell-matrix and cell-cell interactions modulate apoptosis of bronchia epithelial cells. Am f Physiol 1997;272:L28-37.

27 Fine A, Miranda K, Farmer SR, et al. Effect of insoluble extracellular matrix molecules on Fas expression in epithelial cells. F Cell Physiol 1998;174:285-92.

28 DiMango E, Ratner AJ, Bryan R, et al. Activation of NF-kappaB by adherent Pseudomonas aeruginosa in normal and cystic fibrosis respiratory epithelial cells. 7 Clin Invest 1998;101:2598-605.

29 Kashibhata S, Brunner T, Genestier L, et al. DNA damaging agents induce expression of Fas ligand and subsequent agents induce expression of Fas ligand and subsequent kappaB and AP-1. Mol Cell 1998;1:543-51.

30 Rahman I, MacNee W. Role of transcrptional factors in inflammatory lung. Thorax 1998;53:601-12.
31 Tanaka M, Suda T, Haze K, et al. Fas ligand in human serum. Nature Med 1996;2:317-22.

32 Midis G, Shen Y, Owen-Scaub LB. Elevated soluble Fas levels in nonhematopoietic human malignancy. Cancer Res 1996;56:3870-4

33 Taïeb J, Mathurin P, Poinard T, et al. Raised plasma soluble Fas and Fas-ligand in alcoholic liver disease. Lancet 1998;351:1930-1.

34 Suda T, Hashimoto H, Tanaka M, et al. Membrane Fas ligand kills human peripheral blood T lymphocytes, and soluble Fas ligand blocks the killing. f Exp Med 1997;186: 2045-50.

35 Mitsidaes N, Poulaki V, Kotoula V, et al. Fas/Fas ligand up-regulation and BCL-2 down-regulation may be significant in the pathogenesis of Hashimoto's thyroiditis. $f$ Clin Endocrinol Metab 1998;83:2199-203.

36 Kong L, Ogawa N, Nakabayashi T. Fas and Fas L expression in the saliva of patients with primary Sjörgen's syndrome. Arthritis Rheum 1997;40:87-97.

37 Chen JJ, Sun Y, Nabel GJ. Regulation of the proinflammatory effects of Fas ligand (CD95L). Science 1998;282: 1714-7.

38 Hsieh SC, Huang MH, Tsai CY, et al. The expression of genes modulating programmed cell death in normal human polymorphonuclear. Biochem Biophys Res Commun 1997;233:700-6.

39 Chernick WS, Barbero GJ. Composition of tracheobronchial secretions in cystic fibrosis of the pancreas and bronchiectasis. Pediatrics 1959;24:739-45.

40 Shah PL, Scott SF, Knight RA, et al. In vivo effects of recombinant human DNase I on sputum in patients with cystic fibrosis. Thorax 1996;51:119-25. 